\title{
Effect of Stacking Faults on the X-ray Diffraction Profiles of
} $\beta$-SiC Powders

\author{
Vijay V. Pujar* and James D. Cawley \\ Department of Materials Science and Engineering, Case School of Engineering, Case Western Reserve University, \\ Cleveland, Ohio 44106
}

$\mathrm{X}$-ray diffraction patterns of $\beta-\mathrm{SiC}$ (3C or the cubic polytype of $\mathrm{SiC}$ ) powders often exhibit an additional peak at $d=$ $0.266 \mathrm{~nm}$, high background intensity around the (111) peak, and relative intensities for peaks which differ from those predicted from the crystal structure. Computer simulations were used to show that aH these features are due to stacking faults in the powders and not due to the presence of other polytypes in the powders. Such simulations allow diffraction patterns to be generated for different types, frequencies, and spatial distribution of faults. Comparison of the simulation results to the XRD data indicates that the $\beta-\mathrm{SiC}$ particles consist either of heavily faulted clusters distributed irregularly between regions that have only occasional faults or twins, or the powders consist of two types of particles with different populations of faults: those with a high density of faults and those with only twins or occasional faults. Additional information is necessary to determine which description is correct. However, the simulation results can be used to rule out certain fault configurations.

\section{Introduction}

Silicon CARBIDE exists in a number of different polytypic forms. The different polytypes have nearly the same densities, but different crystal symmetries. Although many different polytypes have been reported in the literature, ${ }^{1.2}$ the polytypes $3 \mathrm{C}$ (zincblende structure), $4 \mathrm{H}, 6 \mathrm{H}$, and $15 \mathrm{R}^{\dagger}$ are the most common. In addition, $2 \mathrm{H}$ (wurtzite structure) has been frequently cited as a minor phase. The $3 \mathrm{C}$ polytype is often referred to as $\beta-\mathrm{SiC}$, and all the other polytypes are collectively referred to as $\alpha-\mathrm{SiC}$.

Aspects of the relative stability of polytypes and the conditions under which a given polytype transforms to another are not clear, and this has been a subject of interest for several years (e.g., see Refs. 4-12). The formation of any particular polytype is influenced by the presence of specific impurities and heat treatment. ${ }^{8}$ It is difficult to synthesize single-polytype $\mathrm{SiC}$ powder and it is common for commercially available powders to consist of several polytypes. Also, polytypic transformations can occur in these powders during sintering, of which the $3 \mathrm{C} \rightarrow 6 \mathrm{H}$ transformation is the most common and widely studied..$^{6.13-16}$ Such transformations have a significant influence, often detrimental, on the resulting microstructure and properties, and therefore are of importance in the fabrication of silicon

T. E. Mitchell —contributing editor

Manuscript No. 193617. Received May 4, 1994; approved September 20, 199 Based in part on the thesis submitted by V. V. Pujar for the M.S. degree in Materials Science and Engineering Case Western Reserve University, Cleveland, OH, 1994.

Supported by the NASA-Lewis Research Center, Cleveland, OH, under Grant No. NCC-3-139.

Member, American Ceramic Society.

In the Ramsdell notation, ${ }^{3}$ used for distinguishing between different polytypes, the symbol $n \mathrm{M}$ refers to a polytype with $n$ number of Si-C layers along the $c$-axis of the hexagonal unit cell, and M refers to the cell symmetry, i.e., either cubic $(C)$, hexagonal (H), or rhombohedral (R). carbide for use in engineering applications. ${ }^{13-16}$ Furthermore, the occurrence of polytypic transformations and the nature of the resulting polytype have been shown to be closely related to the presence of stacking faults and the distribution of such faults in the starting material. ${ }^{6-8,13}$ It is therefore desirable to know the type and the relative amounts of different polytypes, and also the type and distribution of stacking faults, present in any given starting material and to monitor the progress of the particular transformations that occur during processing.

The first such studies are those of Jagodzinski (cited in Ref. 17) and Jagodzinski and Arnold, ${ }^{17}$ who attempted a quantitative interpretation of XRD data obtained from powders containing a mixture of polytypes. They were unable to completely describe the patterns assuming any combination of polytypes and attributed the residual mismatch at that time to "unknown disorder effects." They also noted sample-to-sample variations in the number and relative intensities of XRD peaks in different single crystals of nominally the same polytype. In the intervening time, several techniques have been reported in the literature for measuring polytype distributions in $\mathrm{SiC}$ specimens, all of which are essentially variants of a least-squares fit of experimentally observed XRD data to those calculated theoretically for an arbitrarily chosen set of polytypes. Ruska et al. ${ }^{18}$ and Bartram (cited in Ref. 19) calculated the polytype distributions by employing a least-squares fit of intensities of six major peaks in the $30^{\circ}-45^{\circ} 2 \theta$ region assuming that only the $3 \mathrm{C}, 4 \mathrm{H}, 6 \mathrm{H}$, and $15 \mathrm{R}$ polytypes were present in the powders. Since this method involves solving six to eight (depending on the number of peaks used in the $30^{\circ}-45^{\circ} 2 \theta$ range) equations for the unknowns by multiple regression, the results are highly sensitive to moderate fluctuations in the intensities of these peaks. Recently, Frevel et al. ${ }^{19}$ have shown that the accuracy of such calculations can be improved by considering the characteristic nonoverlapping reflections of the noncubic polytypes to determine the concentrations independently (which is essentially an extension of the method originally proposed by Jagodzinski). Attempts have also been made to apply the Rietveld refinement method $^{20}$ for calculating polytype distributions. ${ }^{21}$ Although these techniques yield reliable results for powder mixtures in which each particle is single phase with well-defined lattice parameters, such analyses are generally unreliable in the case of $\mathrm{SiC}$ polytype mixtures for at least two reasons. First, the diffraction patterns from silicon carbide powders contain peaks that are often common to two or more polytypes. Second, and more significant, is the consequence of ubiquitous stacking faults on the relative diffraction intensities and thereby results of polytype distribution obtained from these.

\section{Background}

\section{(1) Polytype Distribution Calculations from XRD Data}

Figure 1 shows a calculated XRD pattern for $3 \mathrm{C}-\mathrm{SiC}$ together with a pattern obtained from a typical $\beta$-SiC powder. ${ }^{\ddagger}$ The two

Ibiden Company, Tokyo, Japan. The data were obtained from a diffractometer

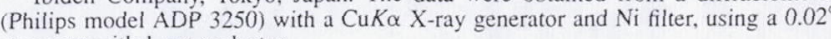
step scan with I s at each step. 
patterns differ in three important ways: the experimental XRD data show an additional peak not associated with the $3 \mathrm{C}$ structure at $2 \theta \approx 33.75^{\circ}(d \approx 0.266 \mathrm{~nm}$, a high background intensity in the region around the (111) peak $(d=0.252 \mathrm{~nm}$, $\left.2 \theta=35.63^{\circ}\right)$; and the $(200)$ peak $\left(d=0.217 \mathrm{~nm}, 2 \theta=41.62^{\circ}\right)$ has a lower relative intensity and is also significantly broader.

The apparent relative amounts of different polytypes in the $\beta$-SiC powder were determined from the XRD data using two different methods. ${ }^{21,22}$ In both, it was assumed that the powders consisted of one or more of the following polytypes: $3 \mathrm{C}, 2 \mathrm{H}$, $4 \mathrm{H}, 6 \mathrm{H}$, or $15 \mathrm{R}$. The results, given in Table I, suggest that the powders contain, in addition to $3 \mathrm{C}$, minor amounts of the other polytypes, notably $2 \mathrm{H}$. Frevel et al. ${ }^{19}$ have also analyzed XRD patterns from $\beta$-SiC powders from the same manufacturer, and their results are also included in Table I. Frevel et al. ${ }^{19}$ attributed the high background intensity in the region around the peak at $d=0.252 \mathrm{~nm}$ to stacking faults (but did not analyze it further) and the occurrence of the non-3C peak (at $d=0.266 \mathrm{~nm}$ ) to minor amounts of other phases, mainly $2 \mathrm{H}$, in the powders. The implication of these analyses is that the powders exist as a mechanical mixture of two types of particles, faulted-3C and $2 \mathrm{H}$ particles. However, such an interpretation is likely incorrect since calculated XRD patterns based on these analyses (Fig. 2) predict the presence of other additional peaks that are not observed in the experimental data; furthermore, they are unable to account for the more or less uniform high background intensity around the (111) peak or changes in relative intensities of peaks.

This paper describes computer simulations that were used to calculate XRD patterns of $3 \mathrm{C}$ structures with prescribed types and distributions of stacking faults. One of the objectives of this work was to confirm that the high background intensity around the peak at $d=0.252 \mathrm{~nm}$ was indeed due to stacking faults in the $3 \mathrm{C}$ structure, and to investigate whether the stacking faults were also responsible for the occurrence of the additional peak

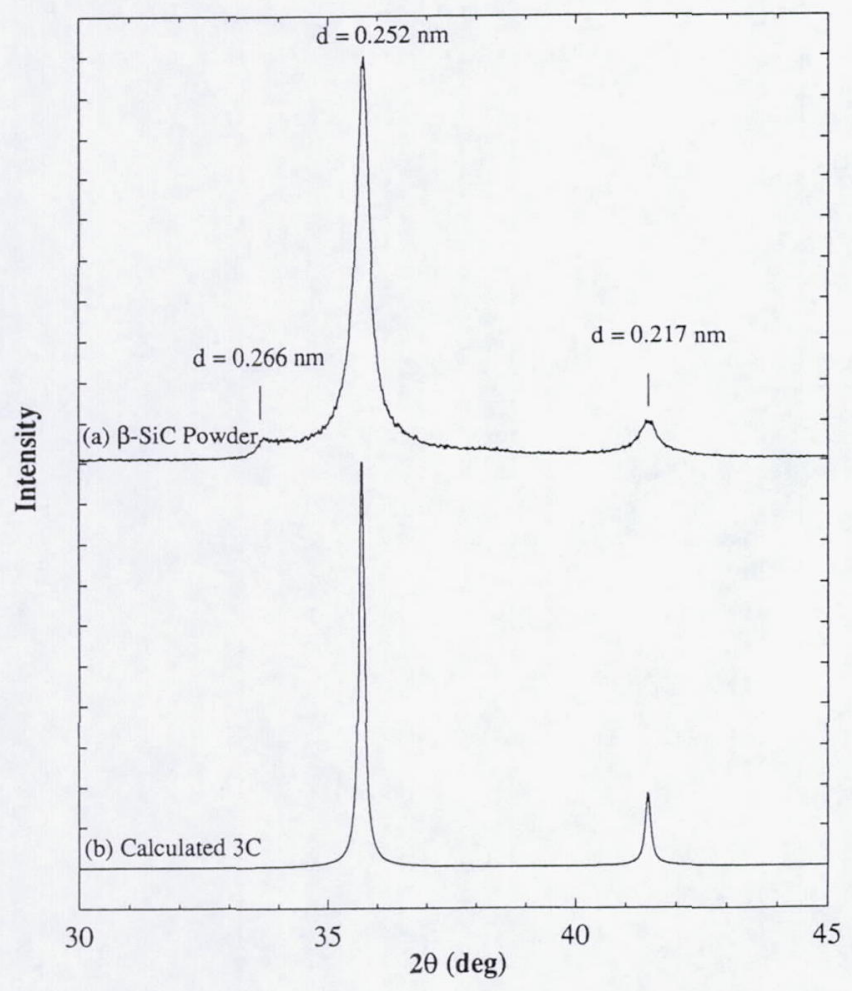

Fig. 1. (a) Experimentally obtained XRD pattern from a commercial $\beta-S i C$ powder, and (b) calculated pattern for a perfect $3 \mathrm{C}$ polytype. at $d=0.266 \mathrm{~nm}$, the changes in relative intensities, and the peak broadening.

\section{(2) Stacking Faults in SiC}

Due to their low formation energies, stacking faults are very common in $\mathrm{SiC}$. Several terminologies are in use to distinguish between different types of faults. These are based on either the origin of the faults or on the spatial arrangement of the faulted sequences relative to the regular sequence, and it is important to clarify the description used in this paper. An ideal facecentered cubic structure can be described as an ... $\mathrm{ABC}$... or equivalently as an ... ACB... stacking sequence of close-packed (111) planes, where the B and C layers are situated above one of the alternate sets of interstices between atoms in the A layers. Thus a cubic crystal results whenever growth occurs such that the new layer is stacked differently with respect to either of the previous two layers. A stacking fault is any error in the regular sequence of layers (with the restriction that adjacent layers are

Table I. Apparent Polytype Distribution (in Volume Percent) in the $\beta$-SiC Powders Obtained Using Different Analysis Methods

\begin{tabular}{lccc}
\hline Polytype & Ruska et al. $^{18}$ & Rietveld method & Frevel et al. $^{19}$ \\
\hline $3 \mathrm{C}$ & 96 & 94.0 & $96 \pm 2$ \\
$2 \mathrm{H}$ & 3 & 2.0 & \\
$4 \mathrm{H}$ & 0 & 0.5 & \\
$6 \mathrm{H}$ & 0 & 0.5 & \\
$15 \mathrm{R}$ & 1 & 1.5 & \\
\hline
\end{tabular}

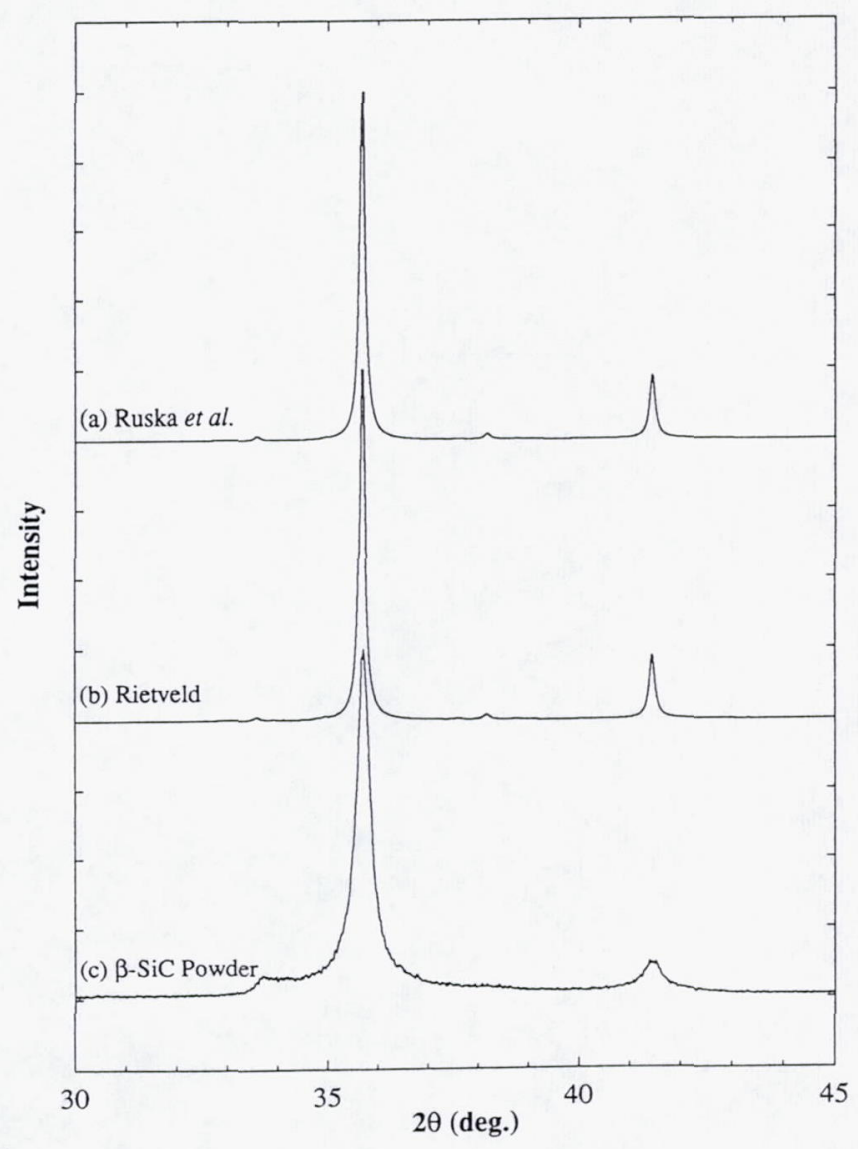

Fig. 2. Calculated XRD patterns based on quantitative analysis of polytype distribution using (a) Rietveld method and (b) Ruska's method. The experimental pattern from the $\beta$-SiC powder (pattern (c)) is included for comparison. In neither case does the pattern calculated using the apparent polytype distribution match the experimental data well. 
always different). In the cubic case, when a fault occurs the new layer is the same as the second-to-last layer. For example, $\mathrm{ABCABACBACB}$ is a sequence with a fault at the sixth layer. The stacking sequence changes from an .... $\mathrm{ABC}$... (positive sequence) to an ... ACB ... (negative sequence) arrangement, or vice versa, at the fault boundary. Since such faults occur commonly during growth, these are sometimes referred to as growth faults; due to the twin relationship between the two portions of the crystal on either side of the fault plane, these are also referred to as twin faults.

A fault could also occur such that the original sequence (either ... $\mathrm{ABC}$... or ... ACB ...) is maintained on either side of the fault plane. For example, $\mathrm{ABCABABCABC...}$ is a sequence with such a fault at the sixth layer. Since such faults occur commonly during deformation, they are often called deformation faults. Alternatively, the sequence $\mathrm{ABCABABCABC}$... may be described as one with a missing layer (the $C$ layer after the fifth layer) at the fault boundary, and analogously, the sequence $\mathrm{ABCABACABC...}$ may be described as one with an extra layer of atoms (an A layer after the fifth layer) at the fault boundary. These configurations are often referred to as, respectively, intrinsic faults and extrinsic faults. Both intrinsic and extrinsic faults fall in the category of deformation faults. Intrinsic and extrinsic faults may also be visualized as the juxtaposition of two twin faults (adjacent layers in the case of intrinsic faults, and alternate layers in the case of extrinsic faults) at the fault boundary. Some researchers prefer to limit the term stacking faults (or sometimes, faults) to mean either intrinsic or extrinsic faults (i.e., deformation faults), while twin faults are referred to as twins. In this paper, we will use the term stacking faults (or faults) to imply an error in the regular sequence. Thus, in this terminology, a single fault results in a twin, two faults in adjacent layers result in an intrinsic fault, and two faults spaced two layers apart result in an extrinsic fault.

The pioneering work by Wilson ${ }^{23,24}$ showed that the presence of stacking faults, specifically twins, in hexagonal close-packed (hcp) structures caused broadening and shifting of certain peaks in XRD data relative to that expected from a material without such faults. Paterson ${ }^{25}$ later extended the Wilson analysis to show that stacking faults in face-centered cubic (fcc) structures also produce peak broadening and lateral shifts in the intensity maxima. In these models, the structure factors and intensity distributions of different peaks were determined as a function of the probability, $\alpha$, for the occurrence of a fault along the $c$-axis of a hexagonal unit cell (in the fcc case, an equivalent hexagonal cell was defined for the purpose of calculating the XRD pattern of the faulted structure). In both models, the procedure for analysis was to define a probability function for different combinations of stacking sequences as a function of $\alpha$ and then calculate the average structure factor, intensity and intensity distribution as a function of this probability. They were able to derive a correlation by which it was possible to predict specific $h k l$ planes that would result in broad or displaced XRD peaks in the faulted structures and estimate the effect of the magnitude of $\alpha$.

Although the models of Paterson and Wilson can, in principle, be used to calculate an entire XRD pattern, the lack of computing machines at the time these models were developed prevented these researchers from making an exhaustive comparison. This limitation, of course, no longer exists. Tateyama et $a l .^{26}$ recently calculated diffraction patterns of faulted $\beta-\mathrm{SiC}$ structures based on the Paterson and Wilson models. Their calculation using the Paterson model predicted the presence of the additional peak at $d=0.266 \mathrm{~nm}$, a high background intensity around the (111) peak $\left(d=0.252 \mathrm{~nm}, 2 \theta=35.63^{\circ}\right)$ and broadening of the $(200)$ peak $\left(d=0.217 \mathrm{~nm}, 2 \theta=41.62^{\circ}\right)$. However, the calculated patterns also showed an increasing lateral shift of the (200) peak toward lower angles with increasing fault densities that did not fit the experimental data. Calculations of diffraction patterns using the Wilson model did not show this shift (even though Wilson's original model does not rule out peak shifts in faulted hcp structures). However, the background intensities around the (111) peak $(d=0.252 \mathrm{~nm})$ were significantly lower than those in the experimental pattern. The background intensity increased with increasing fault densities, but this also resulted in broadening of the (200) peak in excess of that necessary to match the data.

In Wilson's model for hcp metals, a fault in the regular ABAB ... sequence causes a change to either ... ACAC... or BCBC... sequence. Wilson assumed an equal probability for the continuation of either sequence. For the analogous problem in a cubic arrangement, the system differs in that the atoms can be arranged in either of only two equivalent types of arrangement, namely ... ABCABC... (positive) or ...ACBACB ... (negative). The stacking sequence changes from positive to negative, or vice versa, after each occurrence of a fault. Tateyama et $a l .^{26}$ were unable to produce a calculated pattern that closely resembled the experimental pattern when Wilson's model was applied with random faulting. They concluded that it was necessary to introduce correlation between successive faults and expressed the correlation as choosing a faulting pattern such that there were "different continuing probabilities for positive and negative sequences." The best fit to the data resulted when the probability of faults of layers in negative sequences were 3 to 5 times that in the positive sequences. The terms positive and negative have meaning only when the first (positive) has been defined as either ... $\mathrm{ABC}$... or ... $\mathrm{ACB} \ldots$. . Whether the stacking sequence of the parent grain is ... $\mathrm{ABC} \ldots$ or ... ACB ... is immaterial. The physical interpretation of their model then is that the grains have a number of narrow stripes of twinned regions in the crystallites.

Such correlation between stacking faults was also proposed by Jagodzinski ${ }^{6}$ to explain the observation of sharp and diffuse intensity peaks in diffraction data from partially transformed $3 \mathrm{C}$ crystals. He proposed that the transformation occurs by the propagation of 3-layer twins that are separated by integral units of three layers, thus yielding either the perfect $6 \mathrm{H}$ structure or $3 n$-layer stacking faults in $6 \mathrm{H}$. HRTEM studies by Jepps and $\mathrm{Page}^{27}$ on intermediate transformation structures in silicon carbide showed that regions giving rise to diffraction patterns of the type described by Jagodzinski ${ }^{6}$ did indeed show the presence of 3-layer twins that were separated by $3 n$-layers.

In contrast to the Wilson and Paterson approach, the simulations in this paper were carried out using supercells of faulted $3 \mathrm{C}$-SiC structures to study the influence of different faults on the diffraction profiles. Our results complement those of Tateyama et $a .^{26}$ In addition, using supercells allows more direct investigation of the influence of specific types and frequencies of stacking faults on the diffraction profiles. Through these simulations, we investigated the effect of different types and frequencies of faults. The uniqueness of a stacking sequence that reproduces the XRD data is also addressed.

\section{Generation of Diffraction Patterns by Computer Simulation}

The simulations were carried out using supercells that were up to 100 times the volume of a conventional $3 \mathrm{C}$ unit cell Despite the cubic symmetry of the pure polytype, a hexagonal unit cell was defined to simplify the calculation of the atomic positions in the faulted material. The hexagonal unit cell for perfect $3 \mathrm{C}$ (i.e., containing no stacking faults) thus consists of 3 atoms each of $\mathrm{Si}$ and $\mathrm{C}$ arranged in the $\mathrm{ABC}$ sequence along the $z$-axis (Fig. 3). Stacking faults were introduced only in the basal plane of this hexagonal unit cell. This choice is consistent with electron microscopy results, which show that within a given crystallite, all faults usually occur in only one set of parallel close-packed planes (e.g., see Refs. 27 and 28). For computational purposes, this choice allows the two lattice parameters, $a$ and $b$, of the supercell to be held constant while only the lattice parameter $c$ is varied. Thus, the $x$ - and $y$-coordinates defining the position of the atoms are unambiguously defined, depending on whether the atoms belong to the A-, B-, or C-layer. For all atoms in the A-layer, $x=0$ and $y=$ 


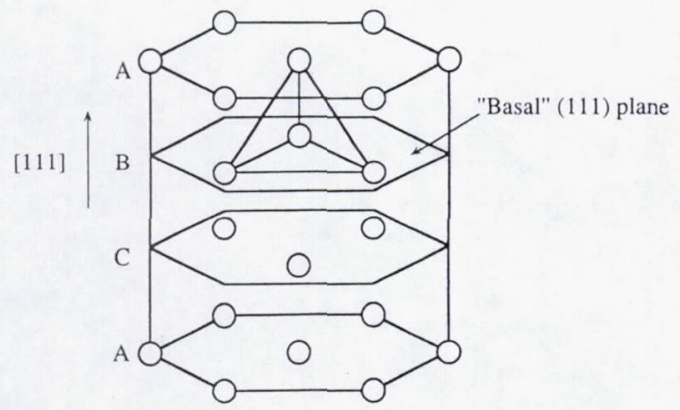

Fig. 3. Hexagonal unit cell representation of atoms in fcc arrangement. The $c$-axis of the unit cell corresponds to a (111) direction in the cubic unit cell.

0 ; for all atoms in the B-layers, $x=1 / 3$ and $y=2 / 3$, and for all atoms in the C-layers, $x=2 / 3$ and $y=1 / 3$, where the coordinates are expressed as a fraction of the corresponding lattice parameter.

The lattice parameter $c$ can be calculated from the number of Si-C double-layer planes, $N$, that are present along the $z$-axis of the unit cell using the relation

$$
c=d_{\mathrm{b}} \cdot N
$$

where the constant $d_{\mathrm{b}}$ is the $d$-spacing of the basal planes. The $z$-coordinate defining the position of the silicon atoms in the $i$ th layer from the bottom of the unit cell is then given by

$$
z_{i}^{\mathrm{Si}}=\frac{c}{N} \cdot i
$$

The corresponding $z$-coordinate of the carbon atoms in the $i$ th layer is given by

$$
z_{i}^{\mathrm{C}}=z_{i}^{\mathrm{Si}}+\delta
$$

where $\delta=0.189 \mathrm{~nm}$ is the $\mathrm{Si}-\mathrm{C}$ bond length. Thus, it is possible to determine the positions of all atoms in the unit cell from the stacking sequence using a straightforward algorithm.
The diffraction patterns were generated using a set of FORTRAN computer programs. The input parameters to the programs include the stacking sequence (specified using the $\mathrm{ABC}$ notation (e.g., ABCABABC, etc.), the lattice parameters ( $a$ and $b)$, the $d$-spacing between the basal planes $\left(d_{\mathrm{b}}\right)$, the $2 \theta$ range over which the XRD data are to be generated, the X-ray wavelength $(\lambda)$, and the standard full-width at half-maximum (FWHM) for the peaks $\left(\Gamma_{\text {std }}\right)$. A simple program calculates the unit cell parameters and the positions of atoms within the cell from the specified $d_{\mathrm{b}}, a, b$, and the stacking sequence. The program also allows more than one unit cell (that is unit cells with different stacking sequences) to be included and is able to calculate the diffraction pattern of such mixtures, when the relative amounts (in volume percent) of these different phases are specified.

The stacking sequence was specified either manually or using the procedure illustrated in the form of a flowchart in Fig. 4. The variables XRAN, YRAN, and ZRAN indicated in the Figure are random uniform deviates between 0.0 and 1.0 generated using a standard subroutine based on three linear congruential generators. ${ }^{29}$ In the sequence generation scheme described in Fig. 3, $P_{1}$ is the probability for the initiation of a fault occurrence in the regular cubic sequence (either twins, or by a small change in the above routine, only intrinsic faults can be specified), $P_{2}$ is the probability that a fault once initiated will be continued, while $P_{3}$ determines the disorder in this faulted region. By selecting appropriate values for $P_{1}, P_{2}$, and $P_{3}$, it is possible to generate a variety of faulted structures ranging from those with a random distribution of single faults (either twins, or intrinsic faults) to those that consisted of clusters of faulted regions. Thus, if $P_{2}$ and $P_{3}$ are zero, then the procedure reverts to the model of Wilson or Paterson, depending on whether twins or intrinsic faults are specified, respectively. With finite $P_{1}$ and $P_{2}$, but $P_{3}=0$, the procedure is equivalent to that employed by Tateyama et al. ${ }^{26}$ Output from the sequence-generating program is illustrated graphically in Fig. 5.

The simulation program first calculates the structure factors, $F_{h k l}$, the $d$-spacing, $d_{h k l}$, and the corresponding scattering angle, $2 \theta$, for $h k l$ indices in the $2 \theta$ region of interest using a preexisting program. ${ }^{30}$ The corresponding intensity, $I$, of the diffracted beam for these $h k l$ planes is then calculated using the simplified relation

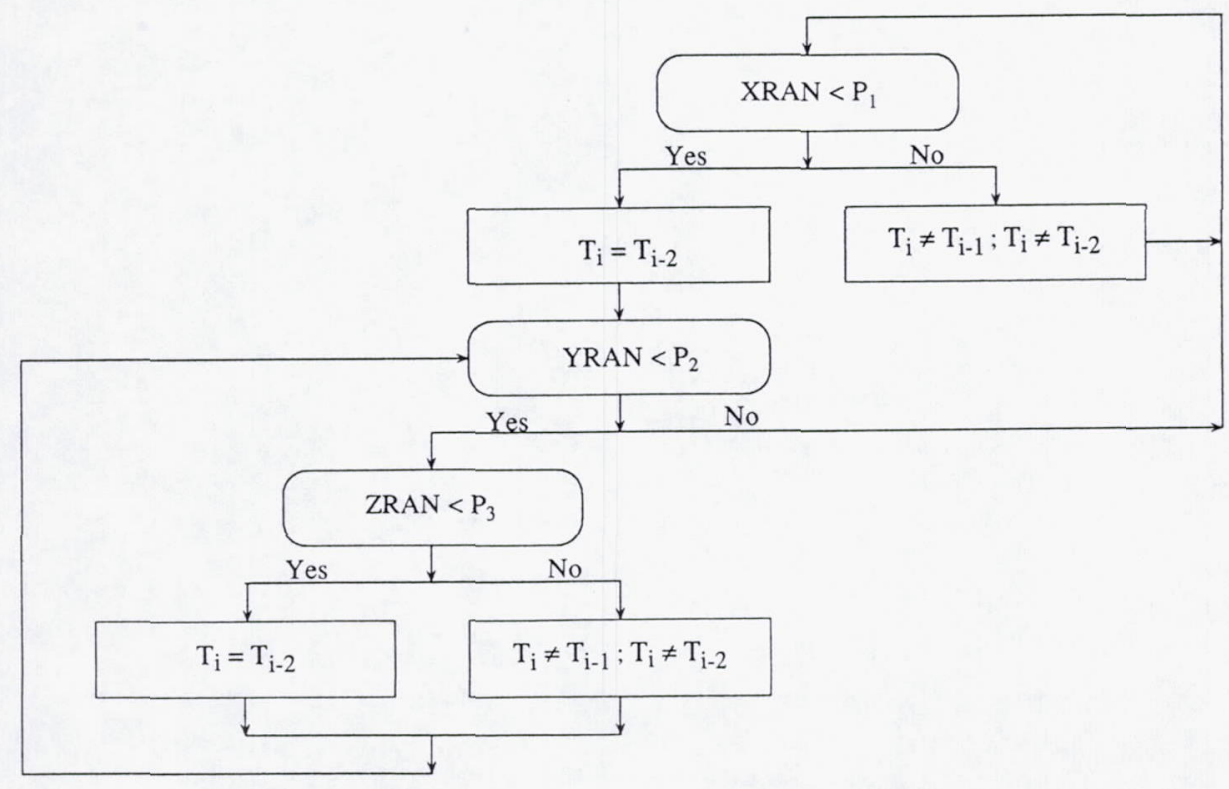

Fig. 4. Flowchart showing the procedure used for generating stacking sequences in the computer simulations. $T_{i}$ refers to the type of a new layer (i.e., either $\mathrm{A}, \mathrm{B}$, or $\mathrm{C}$; thus, $T_{i}=T_{i-2}$ is a formal way of saying that the new layer is identical to the layer that is two layers previous to it). 


$$
I=\left[\frac{|F|}{V}\right]^{2} \cdot \mathrm{LP} \cdot m
$$

where $m$ is the multiplicity factor, and LP is the LorentzPolarization factor, ${ }^{31}$ given by

$$
\mathrm{LP}=\left(\frac{1+\cos ^{2} 2 \theta}{\sin \theta \cdot \cos \theta}\right)
$$

The intensity, $I$, in Eq. (3) is in intensity units per unit volume of the polytype. This normalization makes $I$ independent of the unit cell volume. Thus, for a given stacking sequence, the program returns identical intensities independent of the size of the supercell.

The multiplicity, $m$, is determined by assuming the lowest symmetry conditions, i.e., trigonal symmetry, that is exhibited by all the polytypes and accounting for all the families of $h k l$ planes accordingly (the procedure is outlined clearly in Ref. 32). Structure factors were calculated for all the possible $h k l$
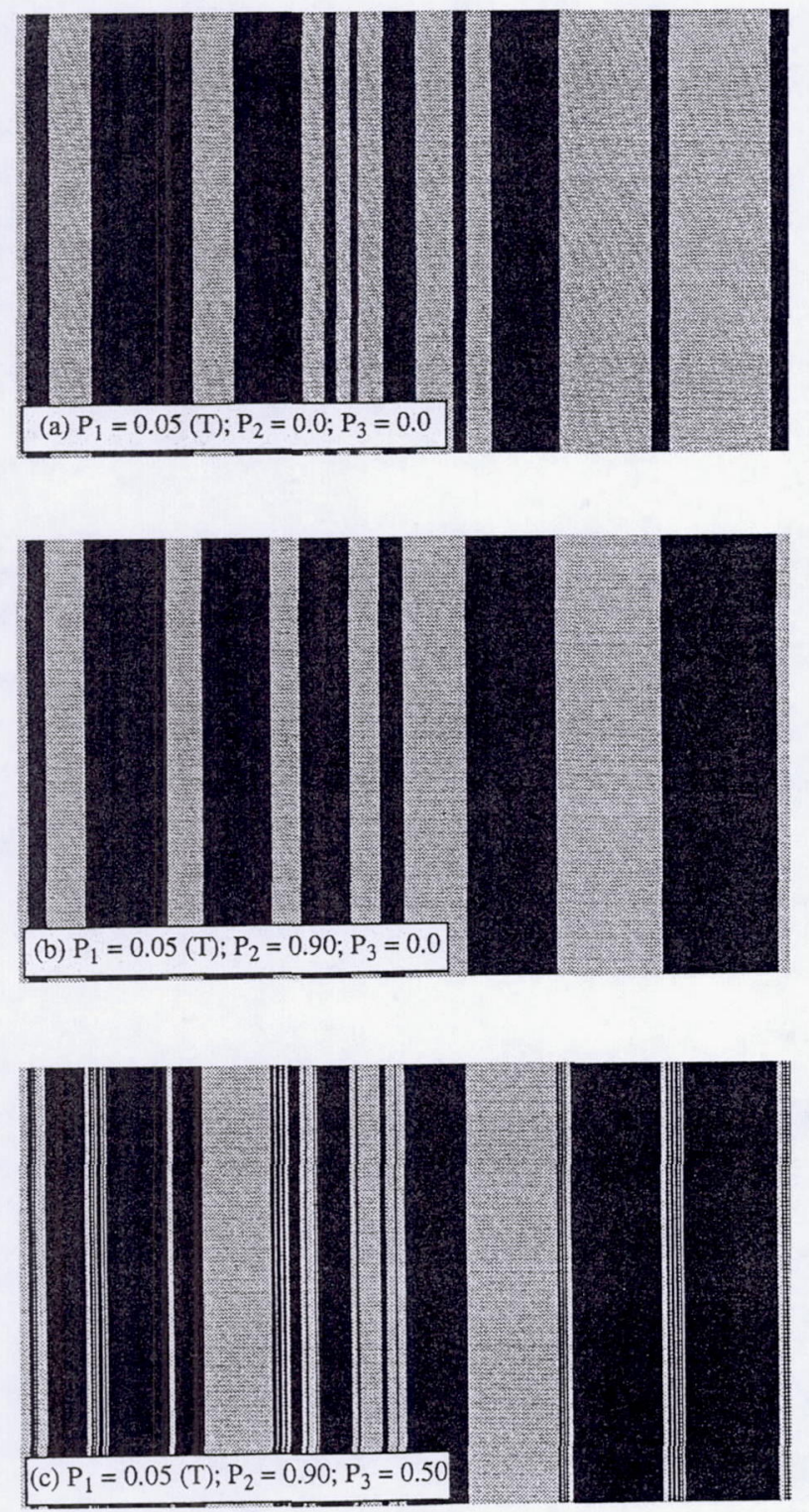

Fig. 5. Graphical illustration of the effect of the three probabilities, $P_{1}, P_{2}$, and $P_{3}$ on the resulting stacking sequences. The dark regions represent stacking in the positive (...ABC...) while the light regions represent stacking in the negative (...CBA ...) directions. When only $P_{1}$ is nonzero, there is a broad distribution in the width of the twinned regions. When $P_{2}$ is also nonzero, a narrower distribution in twin width occurs. The effect of nonzero $P_{3}$ is to form clustered regions of high disorder, i.e., a bimodal fault population results. planes in certain representative cases, and results were compared in order to confirm that the multiplicities were correctly assigned. The latter method of calculating structure factors and thereby intensities, while foolproof, increases computation times unnecessarily.

It was assumed further that the intensity distribution for each peak can be expressed as a Lorentzian function. The total intensity at any given scattering angle, $2 \theta$, can then be determined by summation of the individual contributions of each family of $h k l$ planes that give nonzero intensities within each unit cell for all the $j$ different unit cells, i.e.

$$
I_{2 \theta}=\frac{1}{2 \pi} \sum_{j=1}^{m} \sum_{i=1}^{n} \frac{\Gamma_{\mathrm{std}} \cdot \phi_{j} \cdot I_{i j}}{\left[\left(2 \theta-(2 \theta)_{i j}\right]^{2}+\left(\Gamma_{\mathrm{std}} / 2\right)^{2}\right.}
$$

$I_{i j}$ is the intensity of the $h k l$ family of planes in the $j$ th unit cell and the corresponding scattering angle is $(2 \theta)_{i, j} . \phi_{j}$ is the volume fraction of the polytype whose structure is represented by the $j$ th unit cell and $\Gamma_{\text {std }}$ is the standard FWHM for the peaks.

The diffraction pattern is generated by plotting the intensities, $I_{2 \theta}$, as a function of the scattering angle, $2 \theta$. Although not a limitation of the program itself, the diffraction patterns were usually restricted to the $2 \theta$ range from $30^{\circ}$ to $45^{\circ}$ to minimize computation times. All the major intensity peaks for $\mathrm{SiC}$ lie in this region. Also, for all the simulations, $d_{\mathrm{b}}=0.2517 \mathrm{~nm}$ and $\Gamma_{\text {std }}=0.13^{\circ}$ (the value of $\Gamma_{\text {std }}$ was based on the results of the Lorentzian curve-fit to XRD data from the powders).

\section{Simulation Results}

Figure 6 shows the diffraction patterns for several perfect polytypes generated using the simulation program. The peak intensities in these patterns are in excellent agreement with the reported intensities for these polytypes in the JCPDS. ${ }^{33}$ These results, which are included here for reference, also validate the computer programs.

Figure 7 shows the diffraction patterns calculated for a manually assembled stacking sequence with a single intrinsic stacking fault $(\mathrm{AB})$ occurring at a fixed periodicity of $(\mathrm{ABC})_{n}$ layers. Diffraction patterns were obtained for different values of $n$, namely $n=5,10,20,40$, and 60 . The patterns for the pure polytypes, $2 \mathrm{H}$ (i.e., $n=0$ ) and $3 \mathrm{C}$ (i.e., $n=\infty$ ) are also included in the figure for comparison. In all the cases, the introduction of a stacking fault results in a decrease in the absolute intensities (since only relative intensities are shown, this is not evident from the patterns in Fig. 6) for the peaks associated with perfect $3 \mathrm{C}$. This is due to loss in symmetry and, therefore, a reduced number of equivalent planes (i.e., planes with the same $d$-spacing) contributing to scattering. At the same time, there will be other planes with slightly different $d$-spacing that now result in nonzero intensities. Thus, additional peaks are seen in the diffraction patterns.

As an example, consider the diffraction peak from the $\{111\}$ planes (in the cubic unit cell, $d=0.252 \mathrm{~nm}$ ) in $3 \mathrm{C}$. The equivalent planes on the basis of the hexagonal unit cell are the two $\{0003\}$ basal and half of the twelve $\{10 \overline{1} 1\}$ non-basal planes (since the structure exhibits trigonal symmetry, the other half do not result in diffraction peaks). By introducing an intrinsic stacking fault on the basal plane, the atoms within any basal plane are not disturbed relative to each other. They are, however, displaced in the nonbasal planes. The contribution to the intensity of this peak then, is reduced to that from the basal planes only, and therefore the total intensity is reduced. In the particular case of structures with periodic faults, the original nonbasal planes can result in distinct diffraction peaks, but these will have a different $d$-spacing. The position of these peaks relative to that of the peak at $d=0.252 \mathrm{~nm}$ is dependent on the periodicity of faults. As the periodicity decreases, i.e., $n$ increases, the $d$-spacing between the nonbasal planes converges with the original position. In real powders, the stacking faults are likely to be spaced irregularly and therefore, on an average, yield a weak but more or less uniform intensity in the region 


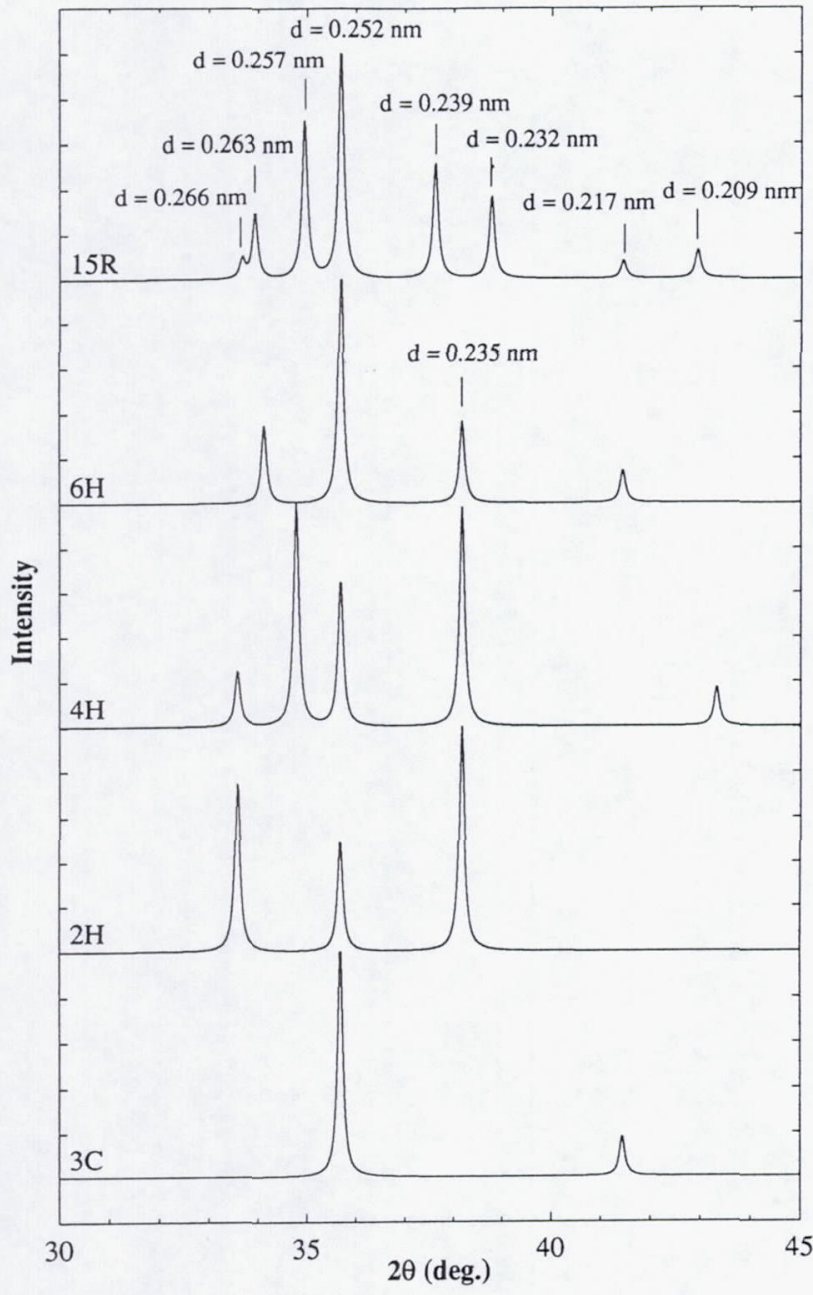

Fig. 6. Calculated patterns for perfect structures of some of the commonly occurring polytypes of $\mathrm{SiC}$.

around the peak at $d=0.252 \mathrm{~nm}$. The patterns in Fig. 6 thus illustrate the physical basis of the enhanced background observed at the base of the (111) peak in the data, although it does not delineate a precise stacking sequence necessary to match the data.

Figure 8 shows diffraction patterns calculated using manually assembled stacking sequences with $2 \mathrm{H}$-type faulted domains present at intervals of 180 layers in cubic arrangement. That is, the stacking sequence in the unit cell can be represented as $(\mathrm{ABC})_{60}(\mathrm{AB})_{m}$. The patterns in the figure correspond to different values of $m$, namely 5,10 , and 20. Again, patterns from perfect $3 \mathrm{C}$ (i.e., $m=0$ ) and $2 \mathrm{H}$ (i.e., $m \approx \infty$ ) are also included for comparison. The height of the peak at $d=$ $0.266 \mathrm{~nm}$ increases with increasing $m$, that is, with increasing thickness of the $2 \mathrm{H}$ domain. Also, a broad peak centered around $d=0.235 \mathrm{~nm}$ is seen, particularly at higher values of $m$. Referring to Fig. 1, it can be seen that both the peak at $d$ $=0.266 \mathrm{~nm}$ and the diffuse peak at $d=0.235 \mathrm{~nm}$ are evident in XRD data from the commercial powder. It is interesting to note in the patterns of Fig. 8 that the peak at $d=0.266 \mathrm{~nm}$ is sharp and relatively strong compared to the peak at $d=0.235 \mathrm{~nm}$ in these patterns, although the latter peak is the strongest for the perfect $2 \mathrm{H}$ polytype.

The above result is consistent with a particle broadening model. ${ }^{31}$ If the $2 \mathrm{H}$ domain is considered as a thin crystallite of $2 \mathrm{H}$ within $3 \mathrm{C}$, then the thickness of the crystallite perpendicular to the planes of interest can be used to estimate the peak broadening expected for each peak,

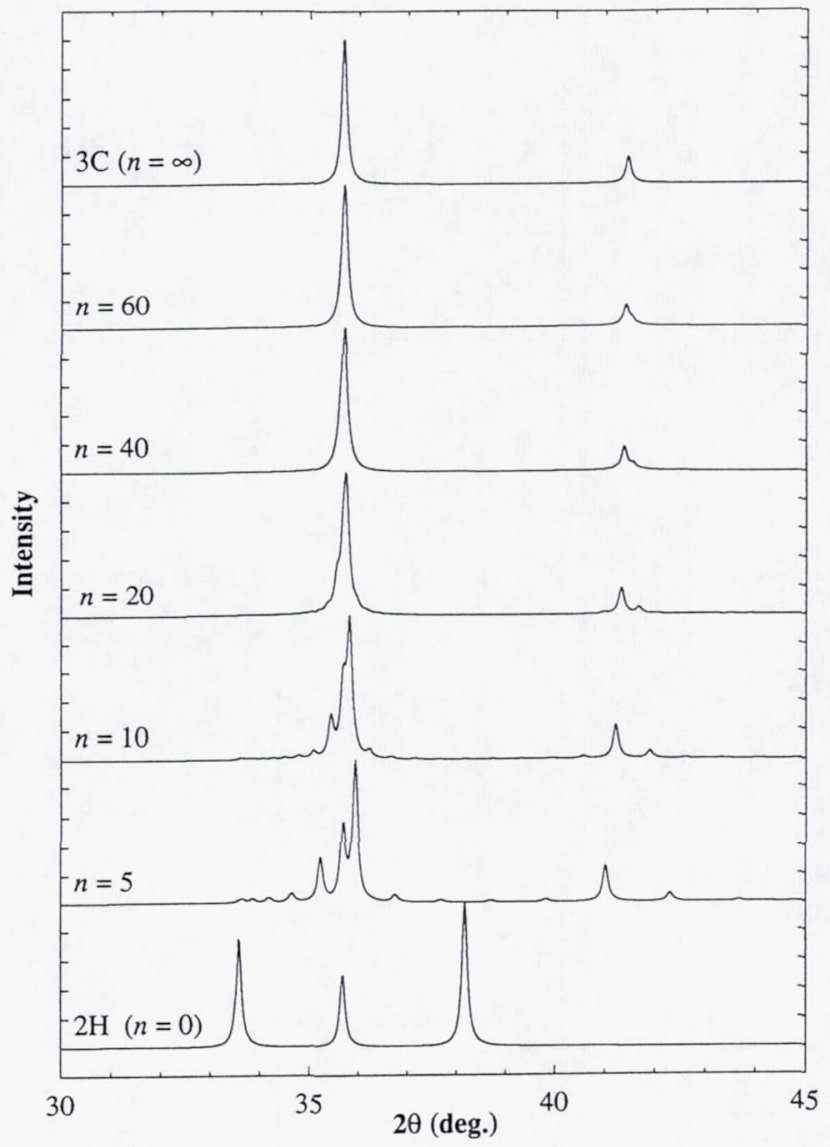

Fig. 7. Diffraction patterns for structures with stacking sequences of the form $(\mathrm{ABC})_{n} \mathrm{AB}$.

$$
t=\frac{0.9 \lambda}{\Gamma_{\mathrm{pb}} \cos \theta}
$$

where $t$ is the effective thickness of the crystallite, $\lambda$ is the $\mathrm{X}$-ray wavelength, $\Gamma_{\mathrm{pb}}$ represents the particle size contribution to the FWHM, and $\theta$ is the Bragg angle. The FWHM values for the peaks can be obtained easily, e.g., by fitting Lorentzian curves in the calculated patterns. Since the $(10 \overline{1} 0)$ planes (which give the peak at $d=0.266 \mathrm{~nm}$ ) are perpendicular to the basal plane, the thickness of the crystallite perpendicular to these planes is of the order of the particle size. Since the simulations assume large particle size, this peak should not experience any particle-size broadening, i.e., $\Gamma_{\mathrm{pb}}=0$. The $\Gamma_{\mathrm{t}}$ value of $0.15^{\circ}$ obtained by fitting the calculated pattern using a Lorentzian is close to the value of $0.13^{\circ}$ assumed for $\Gamma_{\text {std }}$ and therefore consistent with this argument. Using simple geometric relationships, it can be shown that the thickness, $t$, of the $2 \mathrm{H}$

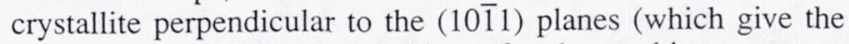
peak at $d=0.235 \mathrm{~nm}$ ) is $5.54 \mathrm{~nm}$ for the stacking sequence $(\mathrm{ABC})_{60}(\mathrm{AB})_{5}$. The predicted value of $\Gamma_{\mathrm{pb}}$ obtained from Eq. (6) is then $1.53^{\circ}$. The total broadening $\Gamma_{t}$ can be estimated using the relation ${ }^{31}$

$$
\Gamma_{\mathrm{t}}^{2}=\Gamma_{\mathrm{std}}^{2}+\Gamma_{\mathrm{pb}}^{2}
$$

$\Gamma_{\text {t }}$ represents the net FWHM that would be observed in the pattern. The resultant value of the predicted value for $\Gamma_{t}, 1.54^{\circ}$, compares very well with the value of $1.5^{\circ}$ deduced for the Lorentzian peak fitted in the calculated pattern. Similarly, the structures with stacking sequences $(\mathrm{ABC})_{60}(\mathrm{AB})_{10}$ and $(\mathrm{ABC})_{60}(\mathrm{AB})_{20}$ give $\Gamma_{\mathrm{t}}=0.80^{\circ}$ and $0.18^{\circ}$, respectively, using Eqs. (6) and (7). These also compare well with the values of $0.73^{\circ}$ and $0.19^{\circ}$ deduced from the calculated pattern. 


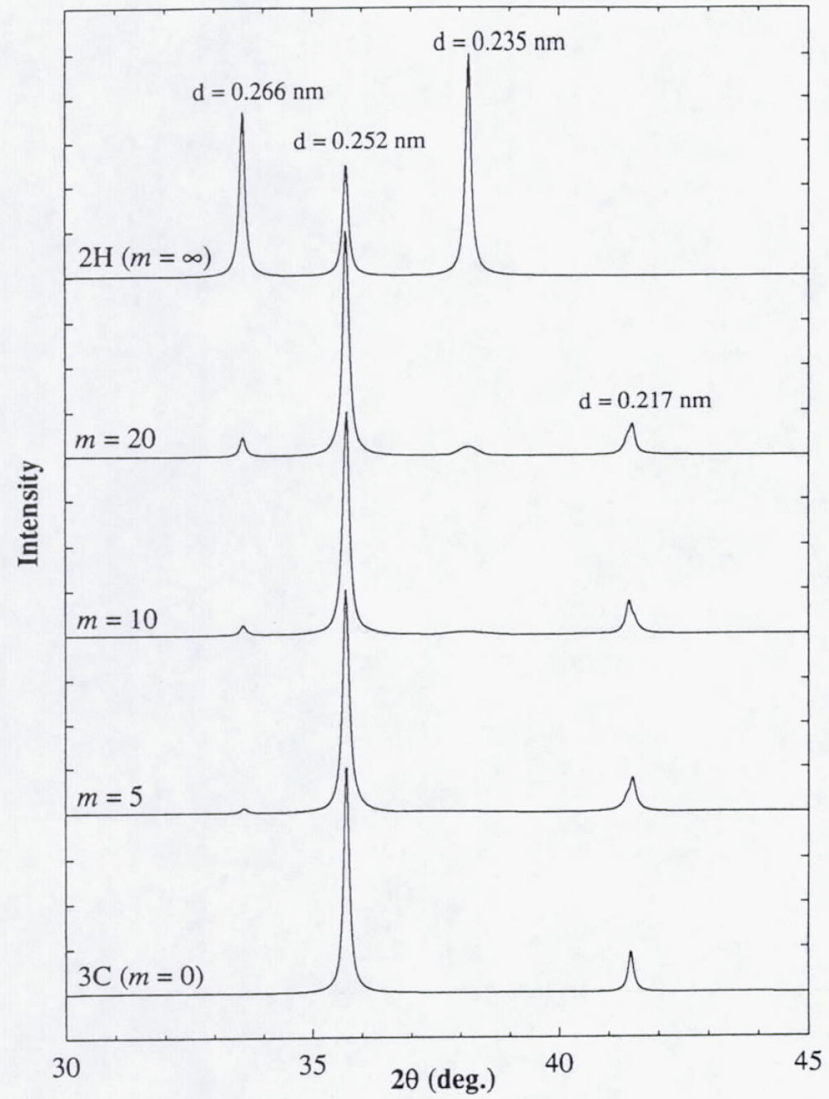

Fig. 8. Diffraction patterns for structures with stacking sequences of the form $(\mathrm{ABC})_{60}(\mathrm{AB})_{m}$.

Simulation runs were also made on large unit cells with 500 layers, the stacking sequences of which were computer generated using the scheme outlined in Fig. 4. By choosing different values for the three probabilities, it was possible to determine the effect of different types of errors in the regular stacking sequence. When both $P_{2}$ and $P_{3}$ were set to zero, the structures were effectively constrained to consist of only twins. For $P_{1}=0.05$, the diffraction pattern exhibited peak broadening and reduction in intensity of the peak at $d=0.217 \mathrm{~nm}$, but did not show the high background intensity around the peak at $d=$ 0.252 observed for the powder patterns (Fig. 9). Increasing the density of twins to $P_{1}=0.20$ resulted in a modest background around the (111), but not sufficient to match the experimental data. It also resulted in such broadening of the (200) peak (at $d=0.217 \mathrm{~nm}$ ) that it is nearly indistinguishable from the background. On the other hand, when the structure contained only intrinsic faults (this was accomplished in the same program by making a small change such that intrinsic faults were always generated, instead of twins), this resulted in a high background intensity, but also resulted in a lateral shift of the peak at $d=0.217 \mathrm{~nm}$, which is not observed in the diffraction pattern from the powder (pattern (c), Fig. 9). Similar to the case of twins, as $P_{1}$ is increased $(\approx 0.2)$, the peak at $d=0.217 \mathrm{~nm}$ diminishes to a broad diffuse peak. Furthermore, the pattern also exhibits a high background intensity around the peak at $d$ $=0.252 \mathrm{~nm}$. All the observed features in the diffraction pattern from the powder are present when both the types of faulted structures are assumed to be present. That is, the simulations suggest that the powders could consist of two kinds of particles which have different fault densities and types: some particles with only $5 \%$ twins and others with a high density (20\%) of intrinsic stacking faults. Figure 11 (pattern (a)) shows the diffraction pattern obtained by averaging results from eight different unit cells; four of them have 5\% twins while the other four have $20 \%$ intrinsic stacking faults, all with independently generated stacking sequences. It can be seen that the pattern is able to reproduce all the features observed in the experimental XRD data.

There are, however, other possible interpretations. As discussed earlier, when the structures have large $2 \mathrm{H}$-type faulted domains, there is no lateral shift in the peak at $d=0.217 \mathrm{~nm}$. These structures can be interpreted as having a low probability of an error occurrence, but are characterized by large faulted domains whenever an error occurs. By generating stacking sequences with a low $P_{1}$ but a relatively high $P_{2}$, and with $P_{3}=$ 1.0 , it is possible to generate sequences with only a small number of thick $2 \mathrm{H}$-type regions within the $3 \mathrm{C}$ crystallite. The diffraction patterns from such structures show a distinct peak at $d=0.266 \mathrm{~nm}$, but the background intensity around the peak at $d=0.252 \mathrm{~nm}$ (Fig. 10(a)) is very low. If we continue to have clustered faults but relax the constraint that these be only of the $2 \mathrm{H}$ type (i.e., letting $P_{3}<1$ ), the observed high background intensity around the peak at $d=0.252 \mathrm{~nm}$ is reproduced. As shown in Fig. 10(b) for a structure with $P_{1}=0.04, P_{2}=0.95$, and $P_{3}=0.5$, the pattern exhibits all the observed features in the pattern. The pattern is characterized, however, by certain additional peaks or shoulders. This is due to an inherent limitation of the simulation in that the pattern represents only a particular type of faulted structure that is periodic after 500 layers. By defining several unit cells with the same values of $P_{1}, P_{2}$, and $P_{3}$, but independently generated random stacking sequences, these will be averaged out as seen in Fig. 11, pattern (b), which represents a composite pattern from five different unit cells with the same values of $P_{1}, P_{2}$, and $P_{3}$. Since a real powder pattern is effectively an average of the patterns of many particles, pattern (b) in Fig. 11 approaches real conditions. The

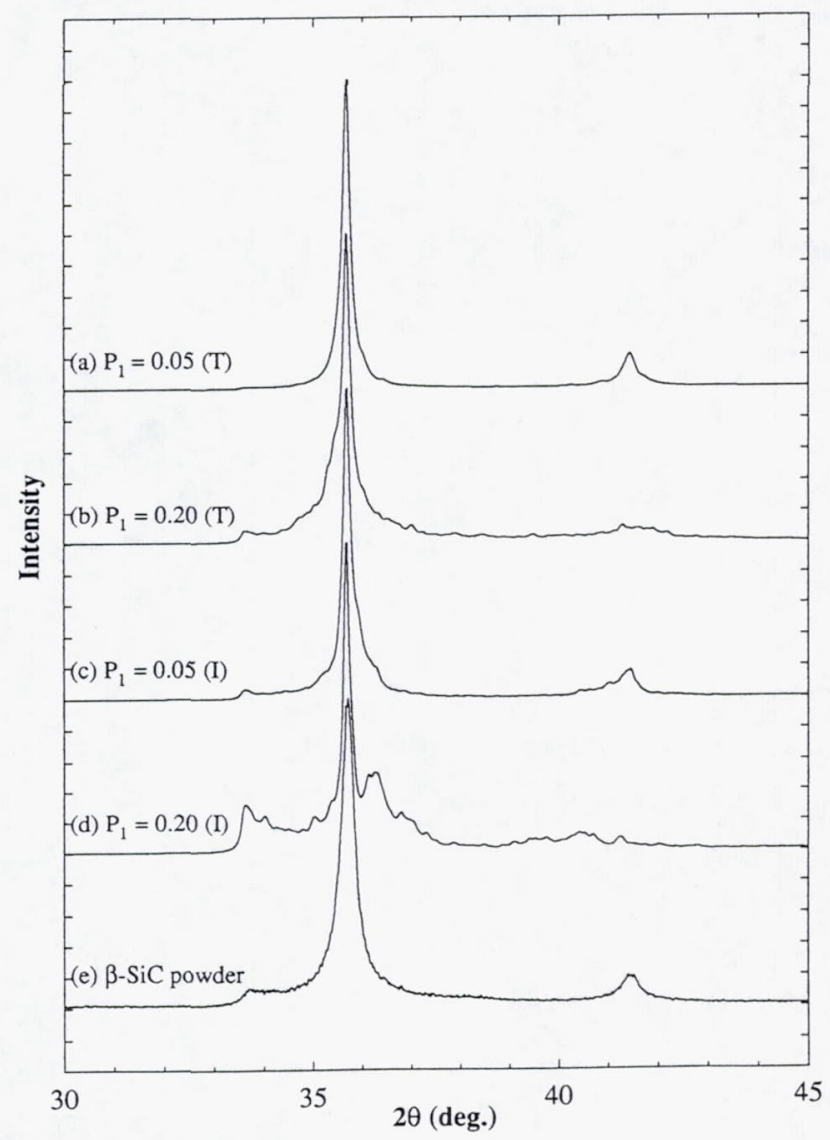

Fig. 9. Diffraction patterns, from 500-layer unit cells with different probabilities, of randomly distributed uncorrelated faults (i.e., $P_{1} \neq 0$, $P_{2}=P_{3}=0$ ): (a) $P_{1}=0.05$ (twins), (b) $P_{1}=0.20$ (twins), (c) $P_{1}$ $=0.05$ (intrinsic faults), and (d) $P_{1}=0.20$ (intrinsic faults). The experimental pattern from the $\beta-\mathrm{SiC}$ powder (pattern (e)) is included in the figure for comparison. 
powder particles that result in this pattern are characterized by regions of regular cubic sequences with only occasional single faults or twins, interspersed with heavily faulted regions that have no particular pattern of faulting. This then represents a second possible scheme of faults in the powders.

This structure with clustered faults is qualitatively similar to the structure deduced by Tateyama et al. ${ }^{26}$ but differs in detail. Their work provides a third independent stacking sequence that also reproduces all the features in the experimental patterns. The calculated pattern based on their scheme of faults is able to reproduce the features observed in that from our $\beta$-SiC powders when the powders are assumed to have 0.075 probability of faults in the positive sequence and 0.30 probability for faults in negative sequence (Fig. 11, pattern (c)).

The spatial distribution of faults in the three possible schemes that are able to independently reproduce the experimental data are schematically illustrated in Fig. 12. These figures illustrate the qualitative differences between the various types of faults and also illustrate the fact that information from the XRD data alone will be inadequate in uniquely describing the type of faults in the powders.

Also, based on these simulations, the sample-to-sample variation in the position and relative intensities of peaks observed by Jagodzinski and Arnold ${ }^{17}$ in XRD data from different single crystals of nominally the same polytype can be attributed to different stacking fault distributions in the individual crystals, thereby resulting in distinctly different patterns.

\section{Summary and Conclusions}

The simulation results show that the presence of stacking faults has a significant influence on the relative intensities of

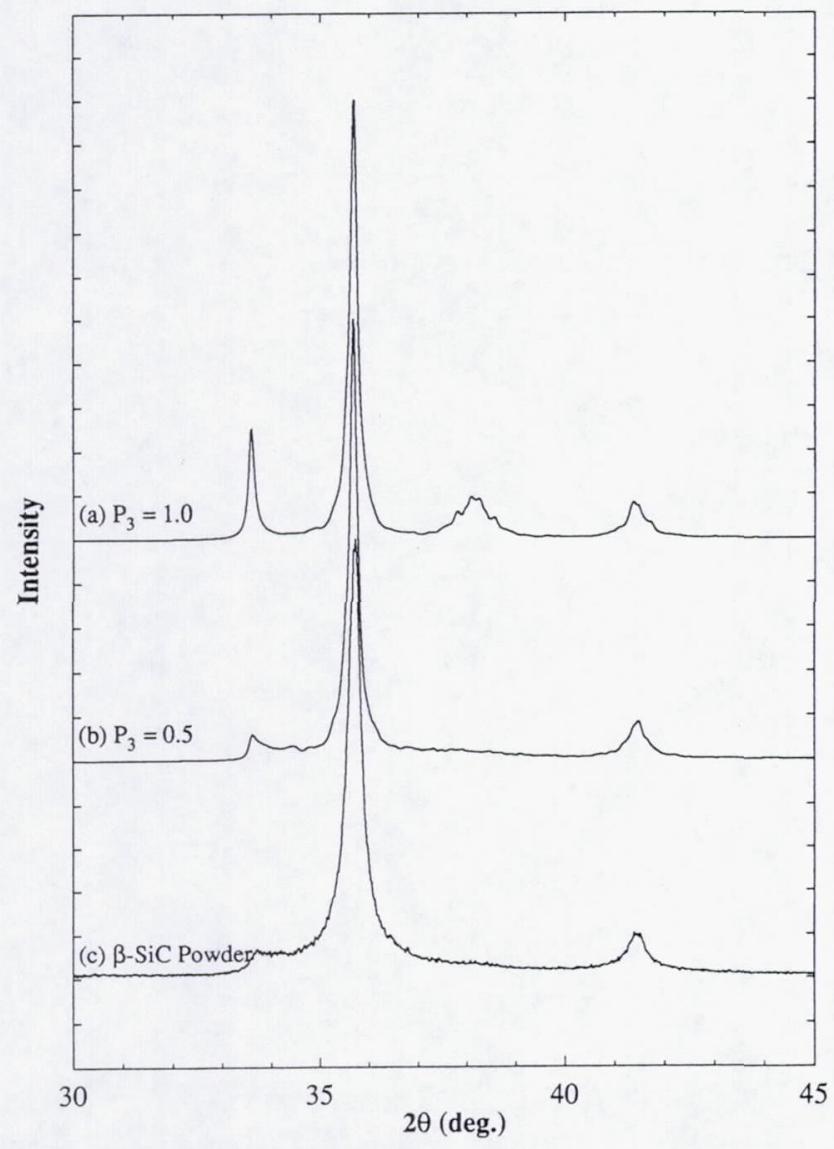

Fig. 10. Diffraction patterns from $3 \mathrm{C}$ structures with fault distributions that were obtained by using $P_{1}=0.04, P_{2}=0.95$, and (a) $P_{3}=$ 1.0 and (b) $P_{3}=0.5$. The experimental pattern from the $\beta-\mathrm{SiC}$ powder (pattern (c)) is again included for comparison.

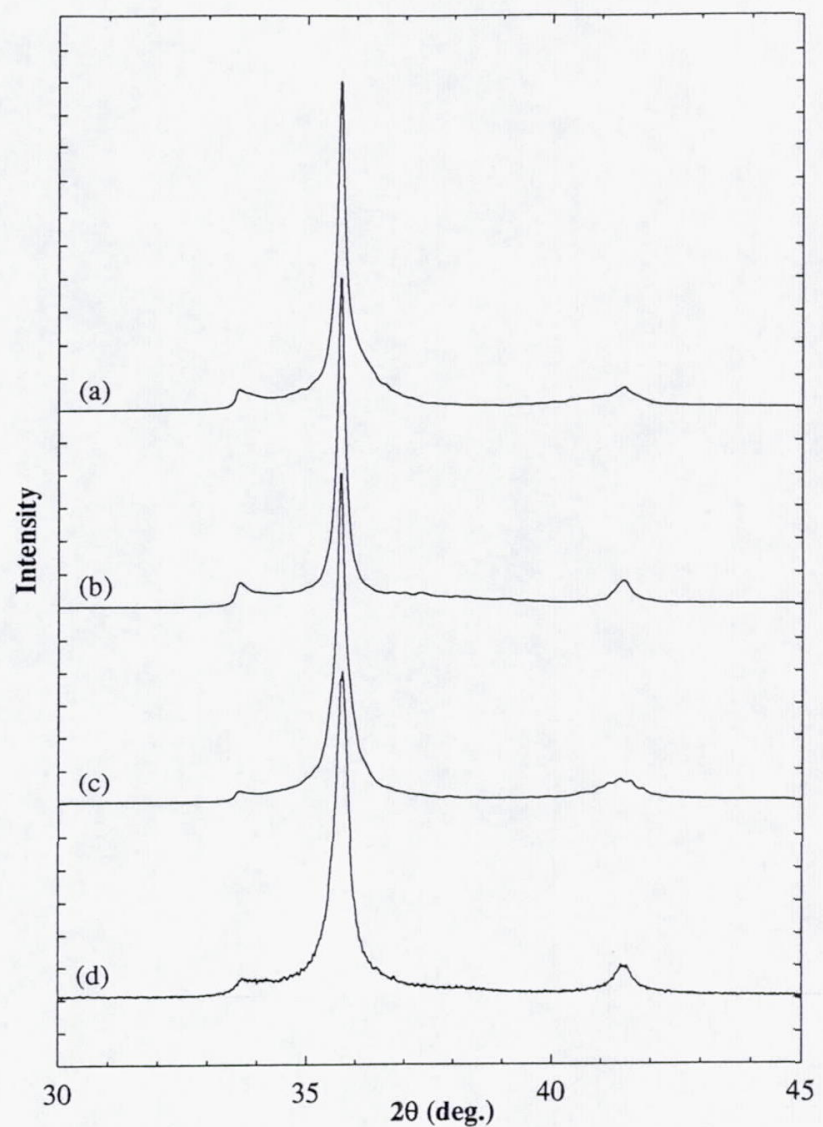

Fig. 11. Diffraction patterns obtained for different types of fault populations. Average patterns (a) from eight unit cells half of which have 5\% twins $\left(P_{1}=0.05, P_{2}=0\right.$, and $\left.P_{3}=0\right)$, and the other half have $20 \%$ intrinsic faults $\left(P_{1}=0.20, P_{2}=0\right.$, and $\left.P_{3}=0\right)$, (b) from five unit cells all with faulted clusters $\left(P_{1}=0.04, P_{2}=0.95\right.$, and $\left.P_{3}=0.5\right)$, and (c) from five unit cells all with 0.075 probability of faults in positive sequence and 0.30 probability of faults in negative sequence. XRD pattern from the $\beta$-SiC powder (pattern (d)) is included in the figure for comparison.

peaks. It has also been shown that all the features observed in the diffraction pattern from $\beta$-SiC powders, namely the presence of an additional peak at $d=0.266 \mathrm{~nm}$, a high background intensity around the peak at $d=0.252 \mathrm{~nm}$, and the broadening of the $3 \mathrm{C}$ peaks, can be explained by the presence of faults in the regular cubic stacking sequences. In the past, the presence of these features has sometimes been attributed to the presence of minor amounts of other polytypes of SiC. First, the peak broadening in the patterns is not at all consistent with the presence of equiaxed $2 \mathrm{H}$ particles. Secondly, the presence of faults changes the relative intensities of the various peaks associated with a given polytype; thus conventional techniques of calculating polytype distributions, which rely entirely on relative intensities of XRD peaks, are unreliable.

The simulations do show that the distribution of faults is not uniformly random. However, they do not provide a unique description of fault population. In addition to the description of faults suggested by Tateyama et al. ${ }^{26}$ (narrow stripes of twins in the crystallites), there are at least two other plausible situations. The $\beta-\mathrm{SiC}$ powders could either consist of two types of particles with different populations of faults and twins, or could consist of a single type of particle that is characterized by clusters of faulted regions.

Acknowledgments: We would like to thank Professors K. P. D. Lagerlöf and A. H. Heuer at CWRU and Dr. R. Teller at BP America Research Center, Cleveland for stimulating discussions. 

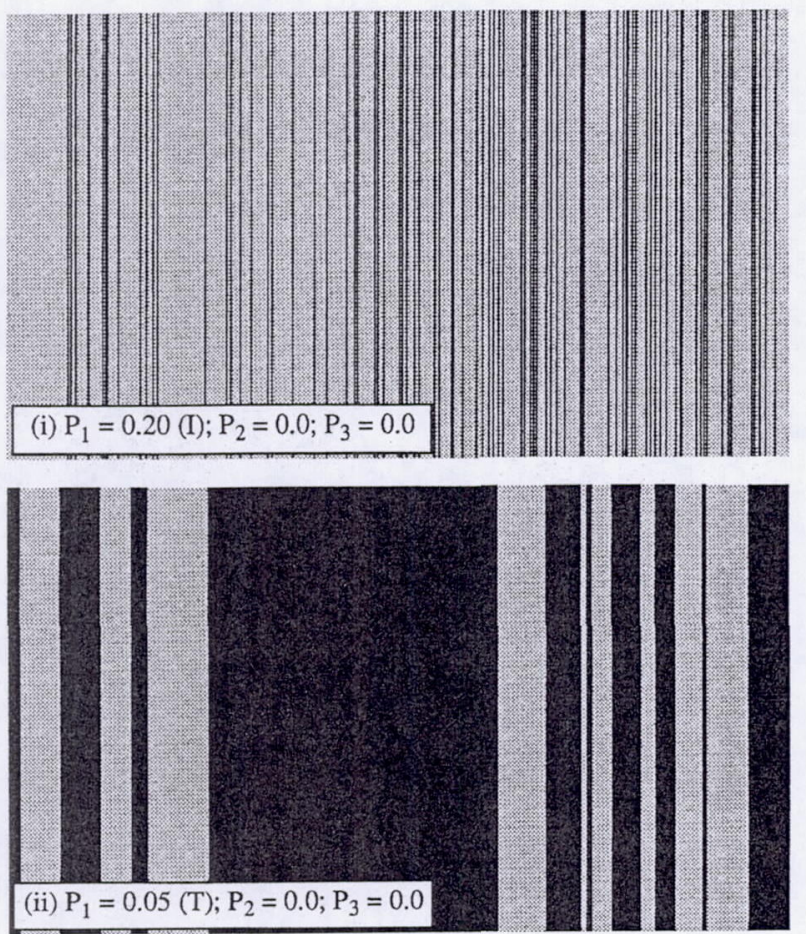

(a)

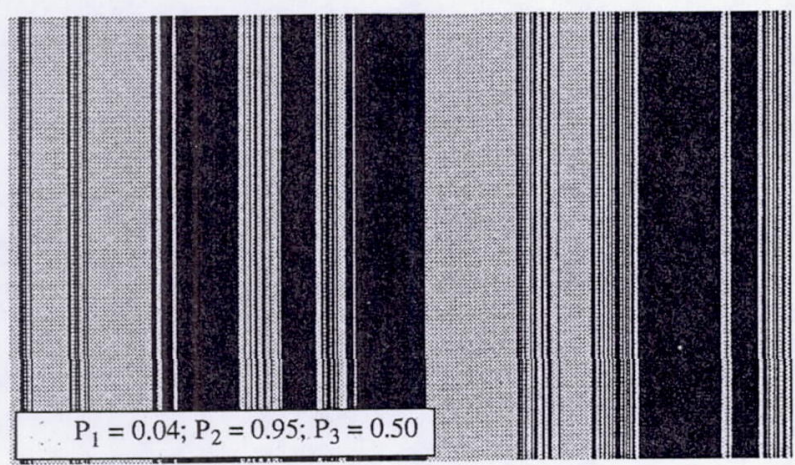

(b)

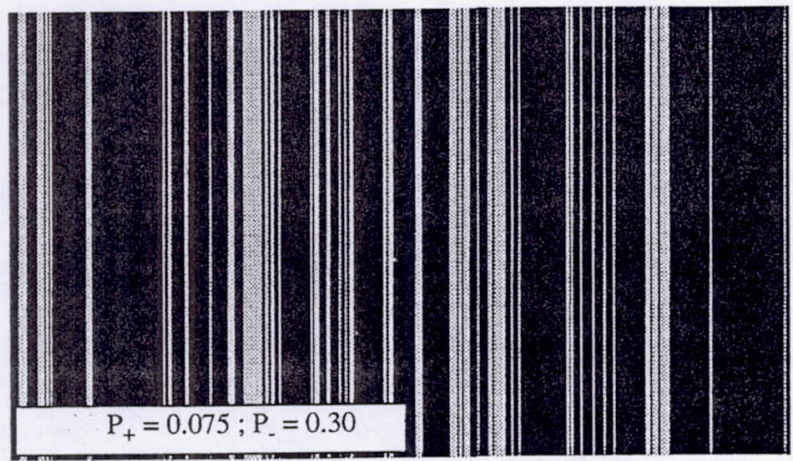

(c)

Fig. 12. Graphical illustration of the different stacking arrangements that are able to independently reproduce all the features observed in the actual pattern: (a) unit cell with (i) $20 \%$ intrinsic faults and (ii) $5 \%$ twins, (b) unit cell with clustered faults $\left(P_{1}=0.04, P_{2}=0.95\right.$, and $P_{3}=0.5$ ), and (c) unit cell with 0.075 probability of faults in positive sequence and 0.30 probability of faults in negative sequence.

\section{References}

'A. R. Verma and P. Krishna, Polymorphism and Polytypism in Crystals; pp. 61-123. Wiley, New York, 1966.

${ }^{2}$ P. T. B. Shaffer, "A Review of the Structure of Silicon Carbide," Acto Crystallogr Sect B. Struct. Crystallogr. Cryst. Chem., 25 [3] 477-88 (1969).

${ }^{3}$ L. S. Ramsdell, "Studies on Silicon Carbide," Am. Miner, 32, 64-82 (1947).

${ }^{4}$ F. C. Frank, "The Growth of Carborundum: Dislocations and Polytypism," Philos. Mag., 42, 1014-21 (1951).

${ }^{5}$ H. Jagodzinski, "Polytypism in Silicon Carbide," Acta Crysallogr., 7, 300 (1954).

${ }^{6} \mathrm{H}$. Jagodzinski, "Transition from Cubic to Hexagonal Silicon Carbide as a Solid-State Reaction," Sov. Phys.-Crystallogr. (Engl. Transl.), 16 [6] 108190 (1972)

'L. U. Ogbuji, "Origin of Long-Period Polytypism in Polycrystalline SiC," Phys. Status Solidi A, 72, 455-61 (1982),

${ }^{8}$ N. W. Jepps and T. F. Page, "Polytypic Transformations in Silicon Carbide," Prog. Cryst. Growth Charact., 7, 259-307 (1983).

${ }^{9} \mathrm{C}$. Cheng, R. J. Needs, and V. Heine, "Inter-Layer Interactions and the Origin of SiC Polytypes," J. Phys. C: Solid State Phys., 21 [1] 1049-63 (1988).

${ }^{10} \mathrm{D}$. Pandey, "The Origin of Stacking Modulations," Phase Transitions, 16/17, 247-61 (1989).

"T. F. Page, "Silicon Carbide: Structure and Polytypic Transformations"; pp 197-214 in The Physics and Chemistry of Carbides, Nitrides and Borides. Edited by R. Freer. Kluwer Academic Publishers, Dordrecht, Netherlands, 1990.

${ }^{12} \mathrm{~V}$. Heine, C. Cheng, and R. J. Needs, "A Computational Study into the Origin of SiC Polytypes," Mater. Sci. Eng., B. 11, 55-60 (1992).

${ }^{13}$ A. H. Heuer, G. A. Fryburg, L. U. Ogbuji, T. E. Mitchell, and S. Shinozaki, " $\beta-\alpha$ Transformation in Polycrystalline SiC: I, Microstructural Aspects," J. Am. Ceram. Soc., 61 [9-10] 406-12 (1978).

${ }^{14}$ L. U. Ogbuji, T. E. Mitchell, A. H. Heuer, and S. Shinozaki, " $\beta \rightarrow \alpha$ Transformation in Polycrystalline SiC: IV, A Comparison of Conventionally

Sintered, Hot-Pressed, Reaction-Sintered, and Chemically Vapor-Deposited Samples," J. Am. Ceram. Soc., 64 [2] 100-105 (1981).

${ }^{15}$ S. Prochazka, "Sintering of Silicon Carbide"; in Mass Transport Phenomena in Ceramics. Edited by A. R. Cooper and A. H. Heuer. Plenum Press, New York, 1975.

${ }^{16}$ S. Prochazka, "Abnormal Grain Growth in Polycrystalline SiC"; pp. 394402 in Proceedings of Third International Conference on Silicon Carbide (Miami, FL, Sept. 1973).

${ }^{17} \mathrm{H}$. Jagodzinski and H. Arnold, "Anomalous Silicon Carbide Structures”; pp. 136-46 in Silicon Carbide (proceedings of conference, Boston, MA, 1959). Pergamon Press, London, U.K., 1960, and references therein.

${ }_{18}^{8}$ J. Ruska, L. I. Gauckler, J. Lorenz, and H. U. Rexer, "The Quantitative Calculation of SiC Polytypes from Measurements of X-ray Diffraction Peak Intensities," J.Mater. Sci.., 14, 2013-17 (1979).

${ }^{19} \mathrm{~L}$. K. Frevel, D. R. Peterson, and C. K. Saha, "Polytype Distribution in Silicon Carbide," J. Mater. Sci., 27, 1913-25 (1992).

${ }^{20} \mathrm{H}$. M. Rietveld, "A Profile Refinement Method for Nuclear and Magnetic Structures," J Appl. Cryst., 2, 65-71 (1969).

${ }^{21} \mathrm{R}$. Teller, BP America, Cleveland, $\mathrm{OH}$, private communication.

${ }^{22} \mathrm{~V}$. V. Pujar, "Microstructural Evolution in SiC-Fibril Reinforced SiC Composites"; M.S. Thesis. Case Western Reserve University, Cleveland, OH, 1994.

${ }^{23}$ A. J. C. Wilson, "Imperfections in the Structure of Cobalt, II. Mathematical Treatment of Proposed Structure," Proc. R. Soc. London, A, 180, 277-85 (1942).

${ }^{24} \mathrm{~A}$. J. C. Wilson, "X-ray Optics: The Diffraction of X-rays by Finite and Imperfect Crystals"; pp. 55-111. Wiley, New York, 1962.

${ }^{25}$ M. S. Paterson, "X-ray Diffraction by Face-Centered Cubic Crystals with Deformation Faults," J. Appl. Phys., 23 [8] 805-11 (1952).

${ }^{26} \mathrm{H}$. Tateyama, N. Sutoh, and N. Murakawa, "Quantitative Analysis of Stack ing Faults in the Structure of SiC by X-ray Powder Refinement Method," J. Ceram. Soc. Jpn. Int. Ed., 96, 985-94 (1988).

${ }^{27} \mathrm{~N}$. W. Jepps and T. F. Page, "Intermediate Transformation Structures in Silicon Carbide," J. Microsc., 119, Pt. 1, 177-88 (1980).

${ }^{28} \mathrm{~L}$. U. Ogbuji, T. E. Mitchell, and A. H. Heuer, " $\beta \rightarrow \alpha$ Transformation in Polycrystalline SiC: III, The Thickening of $\alpha$-Plates," J. Am. Ceram. Soc., 64 [2] 91-99 (1981).

${ }^{29} \mathrm{~W}$. H. Press, B. P. Flannery, S. A. Teukolsky, and W. T. Veterling, "Numerical Recipes-The Art of Scientific Computing"; pp. 196-97. Cambridge University Press, New York, 1986.

${ }^{30}$ K. P. D. Lagerlof, Case Western Reserve University, Cleveland, OH, private communication.

${ }^{3}$ B. D. Cullity, Elements of X-ray Diffraction. Addison-Wesley, Reading, MA, 1978

${ }^{32} \mathrm{~N}$. F M. Henry, H. Lipson, and W. A. Wooster, The Interpretation of X-ray Diffraction Photographs. Macmillan, London, U.K., 1951

${ }^{33}$ Powder Diffraction Files, Card Nos. 29-1126 to 29-1124, and 39-1196. International Centre for Diffraction Data, Newtowne Square, PA. 